\title{
Occurrence of a Mucin-type Glycoprotein in Nidamental Gland Mucosubstance from the Squid Illex argentinus
}

\author{
Shigeru Kimura, ${ }^{* 1}$ Yoko Sugiura, ${ }^{* 1}$ Haruo Mizuno, ${ }^{* 1}$ Noboru Kato, ${ }^{* 2}$ and Yutaka Hanaoka ${ }^{* 2}$ \\ $* 1$ Laboratory of Food Chemistry, Tokyo University of Fisheries, Konan, Minato, Tokyo 108, Japan \\ ${ }^{* 2}$ Kibun Foods Inc., Ginza, Chuo, Tokyo 104, Japan
}

(Received September 16, 1993)

\begin{abstract}
Nidamental glands of the squid Illex argentinus contain mucosubstance which forms jelly-like egg masses after spawning. In this study, a sulfated glycoprotein was prepared from the mucosubstance by solubilization with mild alkaline treatment $\left(0.4 \mathrm{~N} \mathrm{NaOH}, 4{ }^{\circ} \mathrm{C}, 4 \mathrm{~h}\right)$, followed by precipitation with ethanol $(50 \%)$, and was characterized with respect to its chemical composition and macromolecular properties. The glycoprotein accounted for about $35 \%$ of the mucosubstance by weight and comprised $22.6 \%$ protein, $73.0 \%$ carbohydrate, and $4.4 \%$ ester sulfate. A large part of the glycoprotein was isolated after actinase digestion as a long, asymmetric, protease-resistant fragment having a molecular mass of about $1400 \mathrm{kDa}$. The protein backbone of the fragment accounted for $18.7 \%$ by weight and was composed virtually of Thr, Pro, and Ile in the molar ratio of $2: 1: 1$. All Thr residues, comprising $45 \%$ of the total amino acids, were considered to link to GalNAc residues of oligosaccharides consisting mainly of Gal, Fuc, GalNAc, and an unidentified amino sugar. These combined results strongly suggest that the sulfated glycoprotein is heavily $O$-glycosylated and defined as a mucin. The Thr-GalNAc linkages, however, were resistant to $\beta$-elimination $\left(0.5 \mathrm{~N} \mathrm{NaOH}, 25^{\circ} \mathrm{C}, 24 \mathrm{~h}\right)$. The mucin molecules were assumed to be mainly responsible for the viscous and gel-forming properties of the nidamental gland mucosubstance
\end{abstract}

Key words: mucin, nidamental gland, squid, sulfated glycoprotein

Pelagically-spawned eggs of ommastrephid squids such as Illex and Todarodes are wrapped up in fragile gels to form egg masses $50 \mathrm{~cm}$ or larger in diameter. ${ }^{1,2)}$ These gels are secreted by nidamental glands and serve as a physical barrier between the eggs and the surrounding seawater. Furthermore, they appear to trigger sperm release from implanted spermatophores ${ }^{1)}$ and to function as a buoyant mechanism which prevents the eggs from sinking. ${ }^{3)}$

Squid nidamental glands contain gum-like mucosubstance, but its chemical nature has not been studied so far. In this paper, we describe characterization of the main component of the nidamental gland mucosubstance responsible for the formation of egg masses as a mucin-type glycoprotein.

\section{Materials and Methods}

\section{Preparation of Nidamental Gland Mucosubstance}

Frozen samples of the nidamental glands of the squid Illex argentinus were collected at a fish market in Shiogama, Miyagi Prefecture. Each sample weighed $35 \mathrm{~g}$ on average. After thawing in air, the outer membrane of the nidamental glands was dissected with scissors to obtain the mucosubstance.

\section{Preparation of Water-soluble Sulfated Glycoprotein}

About $3 \mathrm{~g}$ of the mucosubstance was homogenized with $150 \mathrm{ml}$ of deionized water, then solubilized with $0.4 \mathrm{~N} \mathrm{NaOH}$ containing $0.1 \mathrm{M} \mathrm{NaBH}_{4}$ for $4 \mathrm{~h}$ at $4^{\circ} \mathrm{C}$ while actively stirring. The resulting viscous solution was clarified by centrifugation at $90,000 \times g$ for $40 \mathrm{~min}$ and treated with $50 \%$ ethanol containing $0.2 \mathrm{M} \mathrm{NaCl}$ in order to precipitate a water-soluble sulfated glycoprotein. The fibrous precipitate thus obtained was easily collected by a pincette and washed completely with $60 \%$ ethanol containing $0.1 \mathrm{M} \mathrm{NaCl}$; if necessary, it was collected by low-speed centrifugation. This precipitate was dissolved in water, dialyzed against large volumes of water, then lyophilized.
Protease Digestion of Water-soluble Sulfated Glycoprotein

The sulfated glycoprotein ( $200 \mathrm{mg}$ ) was dissolved in $200 \mathrm{ml}$ of $0.5 \mathrm{M}$ sodium acetate, $\mathrm{pH} 8.0$, containing $10 \mathrm{~mm} \mathrm{CaCl}_{2}$ and $3 \%$ ethanol, and was digested with $4 \mathrm{mg}$ of actinase (EC $3.4 .24 .4,1 \times 10^{6}$ tyrosine units/g, Kaken Seiyaku Co.), which is a relatively non-specific protease from Streptomyces griseus, for $22 \mathrm{~h}$ at $50^{\circ} \mathrm{C}$. An equal amount of actinase to the first was added after $17 \mathrm{~h}$. Actinase digestion was stopped by adding $\mathrm{NaOH}$ at $4^{\circ} \mathrm{C}$ to a final concentration of $0.1 \mathrm{~N}$, followed by incubation for $10 \mathrm{~min}$, then an actinase-resistant fragment was precipitated with $50 \%$ ethanol. The precipitate was washed with $60 \%$ ethanol containing $0.1 \mathrm{M}$ $\mathrm{NaCl}$, dissolved in water, dialyzed against water, then lyophilized.

\section{Analytical Methods}

Amino acid and amino sugar compositions were determined with amino acid analyzers (Jeol JLC-3BC and Tosoh HPLC system) after hydrolyses of samples in $6 \mathrm{~N} \mathrm{HCl}$ at $110^{\circ} \mathrm{C}$ for $24 \mathrm{~h}$ and in $4 \mathrm{~N} \mathrm{HCl}$ at $100^{\circ} \mathrm{C}$ for $8 \mathrm{~h}$, respectively. Tryptophan was determined after alkaline hydrolysis in the presence of $4 \%$ thiodiglycol. ${ }^{4)}$ Amino sugars were also assayed by the modified Elson-Morgan method ${ }^{51}$ and Ser/Thr-linked GalNAc by the modified Morgan-Elson method. ${ }^{61}$ Neutral sugar composition was determined with a gas chromatograph (Shimazu GC-4C) as trifluoroacetyl derivatives of alditols after hydrolysis in $2.5 \mathrm{~N}$ trifluoroacetic acid at $100^{\circ} \mathrm{C}$ for $7 \mathrm{~h}^{7)}$; arabinose was used as an internal standard. Uronic acids, sialic acids, and ester sulfate were analyzed by the carbazole-sulfuric acid method, ${ }^{8}$ the periodate-resorcinol method, ${ }^{9)}$ and the sodium rhodizonate method, ${ }^{10)}$ respectively. The $N$-acetyl groups of amino sugars were determined on a TSKgel OApak column (Tosoh) as acetic acid liberated by hydrolysis in $2 \mathrm{~N} \mathrm{HCl}$ at $100^{\circ} \mathrm{C}$ for $2 \mathrm{~h}$. The lipid and ash contents were determined by the usual methods.

The sedimentation velocity was analyzed with an analytical ultracentrifuge (Hitachi model 282) at $50,000 \mathrm{rpm}$ and at $25.0^{\circ} \mathrm{C}$. Samples were dissolved in $0.6 \mathrm{M} \mathrm{NaCl}$ containing $0.02 \mathrm{M}$ sodium phosphate, pH 7.2, at several concentrations between 0.018 and $0.045 \%$. The values of $S_{20, w}^{\circ}$ was calculated using a partial specific volume of $0.64 \mathrm{~m} / \mathrm{g}$ obtained from a comparison of published values of a range of glycoproteins. ${ }^{11}$ Viscosity was measured on $5 \mathrm{~m} /$ sample solutions with a Cannon-Fenske viscometer having an average shear gradient of about $1000 \mathrm{~s}^{-1}$. Electrophoresis in sodium dodecyl sulfate (SDS) was carried out on $3.5 \%$ polyacrylamide gels containing $0.1 \%$ SDS and $3.5 \mathrm{M}$ urea. ${ }^{12)}$ Protein bands were visualized 
with Coomassie Brilliant Blue R-250, and molecular weight markers (Sigma) for SDS-gel electrophoresis were used as standard proteins. Infrared spectra were obtained with a spectrophotometer (JEOL JIR-RFX3002) in $\mathrm{KBr}$ pellets.

\section{Results}

\section{Mucosubstance from Squid Nidamental Glands}

The mucosubstance from the nidamental glands of Illex argentinus was very sticky and its water content was about $76 \%$ by weight. Chemical analyses showed that it comprised a large number of constituents; $48.6 \%$ crude protein, $37.1 \%$ carbohydrate, $6.9 \%$ lipid, $5.5 \%$ ash, and $1.9 \%$ sulfate by dry weight. The amino acid, sugar, and sulfate compositions are listed in Table 1, in terms of residues per 1000 total amino acid and sugar residues. Proteins of the mucosubstance were relatively rich in threonine and aspartic acid. As for carbohydrates, galactose, fucose, and $N$-acetylga-

Table 1. Chemical compositions of squid nidamental gland mucosubstance and sulfated glycoprotein (mucin) (residues/1000 total amino acid and sugar residues)

\begin{tabular}{|c|c|c|c|}
\hline & \multirow[b]{2}{*}{ Mucosubstance } & \multicolumn{2}{|c|}{ Sulfated glycoprotein (mucin) } \\
\hline & & Whole & $\begin{array}{l}\text { Actinase-resistant } \\
\text { fragment }\end{array}$ \\
\hline \multicolumn{4}{|l|}{ Amino acid } \\
\hline Tau & 26 & 0 & 0 \\
\hline Asp & 65 & 13 & 3 \\
\hline Thr & 74 & 125 & 132 \\
\hline Ser & 28 & 4 & 1 \\
\hline Glu & 54 & 10 & 3 \\
\hline Pro & 57 & 65 & 68 \\
\hline Gly & 51 & 10 & 5 \\
\hline $\mathrm{Ala}$ & 34 & 6 & 1 \\
\hline Cys $/ 2$ & 23 & 0 & 0 \\
\hline Val & 36 & 11 & 6 \\
\hline Met & 8 & 1 & 1 \\
\hline Ile & 48 & 63 & 65 \\
\hline Leu & 35 & 8 & 2 \\
\hline Tyr & 21 & 3 & 1 \\
\hline Phe & 21 & 4 & 1 \\
\hline Trp & 3 & 0 & 0 \\
\hline Lys & 51 & 11 & 4 \\
\hline His & 14 & 2 & 1 \\
\hline Arg & 22 & 5 & 2 \\
\hline Total & 671 & 341 & 296 \\
\hline \multicolumn{4}{|c|}{ Neutral sugar } \\
\hline Rha & 24 & 52 & 62 \\
\hline Fuc & 59 & 122 & 107 \\
\hline $\mathrm{Rib}$ & 13 & 16 & 26 \\
\hline Xyl & 24 & 35 & 49 \\
\hline Man & 19 & 6 & 7 \\
\hline Glc & 20 & 34 & 14 \\
\hline Gal & 72 & 167 & 190 \\
\hline Total & 231 & 432 & 455 \\
\hline \multicolumn{4}{|c|}{ Amino sugar } \\
\hline GlcNAc & 19 & 33 & 31 \\
\hline $\mathrm{X}^{*}$ & 36 & 78 & 88 \\
\hline GalNAc & 43 & .116 & 130 \\
\hline Total & 98 & 227 & 249 \\
\hline Total & 1000 & 1000 & 1000 \\
\hline $\mathrm{SO}_{4}^{2-}$ & 32 & 72 & 81 \\
\hline
\end{tabular}

* An unidentified amino sugar was determined as a glucosamine equivalent. lactosamine were recognized to be the major components, while uronic acids and sialic acids were not detected.

Of particular interest is the occurrence of a considerable amount of unidentified amino sugar (designated X). As shown in Fig. 1, there are three distinct amino sugars, glucosamine, $\mathrm{X}$, and galactosamine, in the order of their elution positions, which are positive for the modified Elson-Morgan reaction. ${ }^{5)}$ In contrast with glucosamine and galactosamine, complete loss of the $\mathrm{X}$ component was seen in an experiment in which the mucosubstance was hydrolyzed by strong acid hydrolysis $\left(6 \mathrm{~N} \mathrm{HCl}, 110^{\circ} \mathrm{C}, 24 \mathrm{~h}\right)$. Although the glucosamine and galactosamine were destroyed too much by the $6 \mathrm{~N} \mathrm{HCl}$ to give accurate values, the ratio of glucosamine to galactosamine increased significantly (results not shown) compared with that in other experiments in which the sample was hydrolyzed to obtain amino sugar data $\left(4 \mathrm{~N} \mathrm{HCl}, 100^{\circ} \mathrm{C}, 8 \mathrm{~h}\right)$. It is likely that some degradation products of $\mathrm{X}$ were eluted from the amino acid analyzer at the position corresponding to glucosamine; a structural similarity between glucosamine and $\mathrm{X}$ may exist. Thus the X component was tentatively determined as a

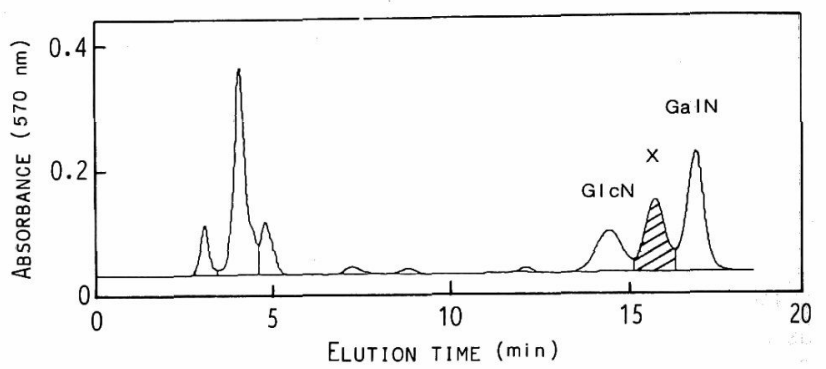

Fig. 1. Chromatographic separation of an unidentified amino sugar (designated as $\mathrm{X}$ ) from squid nidamental gland mucosubstance on an amino acid analyzer.

The hydrolyzed sample was eluted from a TSKgel Aminopak

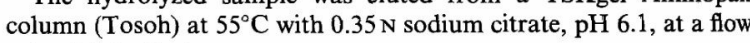
rate of $0.4 \mathrm{ml} / \mathrm{min}$.

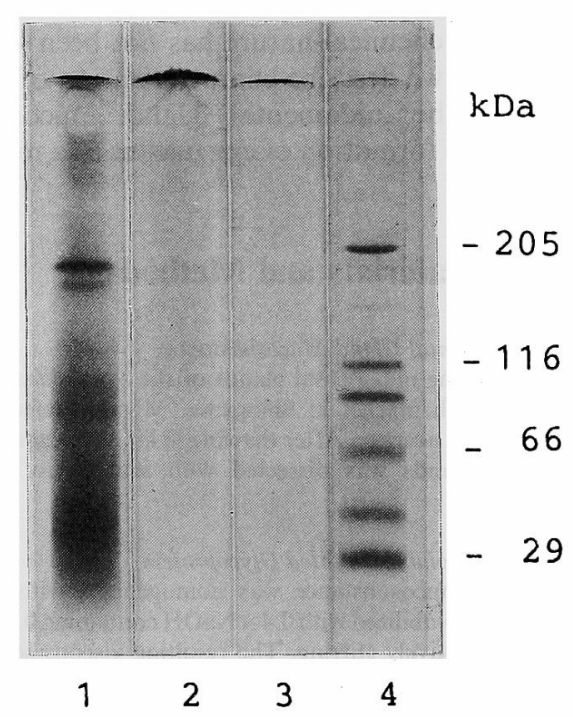

Fig. 2. SDS-polyacrylamide gel electrophoresis of nidamental gland mucosubstance and sulfated glycoprotein.

Each gel was visualized with Coomassie Brilliant Blue R-250. 1, mucosubstance; 2, glycoprotein; 3, actinase-resistant fragment of glycoprotein; and 4, SDS molecular weight markers (Sigma). 
glucosamine equivalent. Furthermore, this component, as well as glucosamine and galactosamine, was found to be $\mathrm{N}$-acetylated, because the equimolar amounts of acetic acid and amino sugar were obtained after acid hydrolysis of the mucosubstance.

The nature of the proteins in the mucosubstance was also assessed by $3.5 \%$ polyacrylamide gel electrophoresis in SDS (Fig. 2). The electrophoretic pattern revealed a protein band which remained at the gel top, two distinct bands having molecular masses of about 170 to $190 \mathrm{kDa}$, and fuzzy bands in the molecular mass range of 20 to $110 \mathrm{kDa}$.

\section{Sulfated Glycoprotein from Nidamental Gland Muco- substance}

Most of the mucosubstance was easily solubilized with $0.4 \mathrm{~N} \mathrm{NaOH}$ at $4^{\circ} \mathrm{C}$ to give a highly viscous solution. After the alkaline treatment for $4 \mathrm{~h}$, a fibrous precipitate was produced from the viscous solution by adding ethanol to a final concentration of $50 \%$. The precipitate was found to be a water-soluble sulfated glycoprotein comprising $22.6 \%$ protein, $73.0 \%$ carbohydrate, and $4.4 \%$ sulfate by weight. The yield was about $35 \%$ of the mucosubstance by dry weight. Moreover, electrophoresis in SDS shows that the sulfated glycoprotein does not enter the $3.5 \%$ polyacrylamide gel (Fig. 2). As listed in Table 1, the protein fraction of sulfated glycoprotein is characterized by high levels of threonine, proline, and isoleucine. Particularly, it is noteworthy that threonine accounted for one-third of the total amino acid residues, because this amino acid is assumed to be the main candidate for protein-carbohydrate likages in this glycoprotein. The carbohydrate fraction contained galactose, fucose, $\mathrm{N}$-acetylgalactosamine, and $\mathrm{X}$ as the major components and several sugars such as rhamnose, ribose, and so on as the minor ones. The amount of $N$-acetylgalactosamine, 116 residues $/ 1000$, was very similar to that of threonine, 125 residues $/ 1000$; these data clearly indicate the possible existence of $O$-glycosidic linkages between threonine and $N$-acetylgalactosamine residues which are typical of mucin-type glycoproteins. ${ }^{13}$ ) The lack of appreciable change in the threonine content of the sulfated glycoprotein, however, was observed after treatment with $0.5 \mathrm{~N} \mathrm{NaOH}$ at $25^{\circ} \mathrm{C}$ for $24 \mathrm{~h}$, suggesting that $\beta$-elimination reaction did not occur significantly. Moreover, the sulfated glycoprotein gave no detectable color (due to Kuhn's chromogens) using the modified Morgan-Elson assay method.

The content of ester sulfate was 72 residues/1000 which were presumably linked to sugar components such as galactose, $\mathrm{N}$-acetylgalactosamine, or $\mathrm{N}$-acetylglucosamine. ${ }^{13)}$ In this connection, the infrared spectrum of sulfated glycoprotein has absorption at $1240 \mathrm{~cm}^{-1}$ and $820 \mathrm{~cm}^{-1}$, indicating the existence of equatorial sulfate groups (Fig. 3).

The sulfated glycoprotein was very soluble in water and the highly viscous solution thus obtained was examined by viscometry. Figure 4 shows the changes in reduced viscosity of $0.025 \%$ solution at three different concentrations of $\mathrm{NaCl}$. An extremely high viscosity, $100 \mathrm{~d} l / \mathrm{g}$, in the absence of $\mathrm{NaCl}$ dropped sharply to about one-seventh of the original value even at a low concentration of $\mathrm{NaCl}(0.02 \mathrm{M})$, followed by a gradual decrease of the viscosity to $0.6 \mathrm{M}$ $\mathrm{NaCl}$. Moreover, the intrinsic viscosities were determined to be $40.0 \mathrm{~d} l / \mathrm{g}$ in water and $10.0 \mathrm{~d} l / \mathrm{g}$ in $0.6 \mathrm{M} \mathrm{NaCl}$

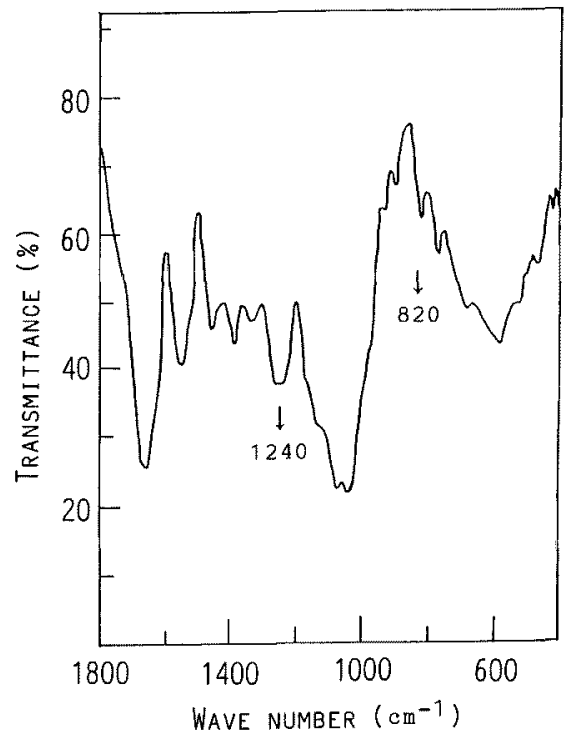

Fig. 3. Infrared spectrum of nidamental gland glycoprotein.

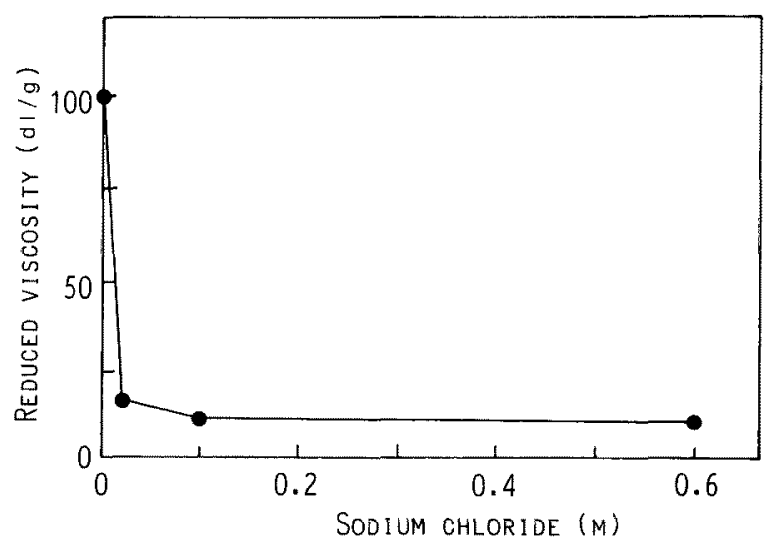

Fig. 4. Effects of $\mathrm{NaCl}$ on reduced viscosity of nidamental gland glycoprotein.

The glycoprotein was dissolved in water at $25^{\circ} \mathrm{C}$ and after addition of $\mathrm{NaCl}$, viscosity was measured at a glycoprotein concentration of $0.025 \%$

containing $0.02 \mathrm{M}$ phosphate buffer, $\mathrm{pH} 7.2$, as shown in Fig. 5. In these experiments, the glycoprotein solutions were made slightly turbid in the presence of $\mathrm{NaCl}$; some molecular aggregations must have occurred. In the case of water as the solvent, Fig. 5 also shows the strong dependency of reduced viscosity on glycoprotein concentration. This could be explained by the absence of charge shielding in water, which allows increased repulsion among the negative charges of ester sulfate residues and thus increased expansion and asymmetry of the glycoprotein molecule.

\section{Protease-resistant Fragment of Sulfated Glycoprotein}

The proteolytic reaction mixture was incubated for $22 \mathrm{~h}$ at $50^{\circ} \mathrm{C}$ then treated with ethanol to precipitate actinaseresistant large fragments. Approximately $85 \%$ of the original weight of sulfated glycoprotein was collected by $50 \%$ ethanol precipitation; low molecular weight fragments released by the actinase were found to be soluble in the 


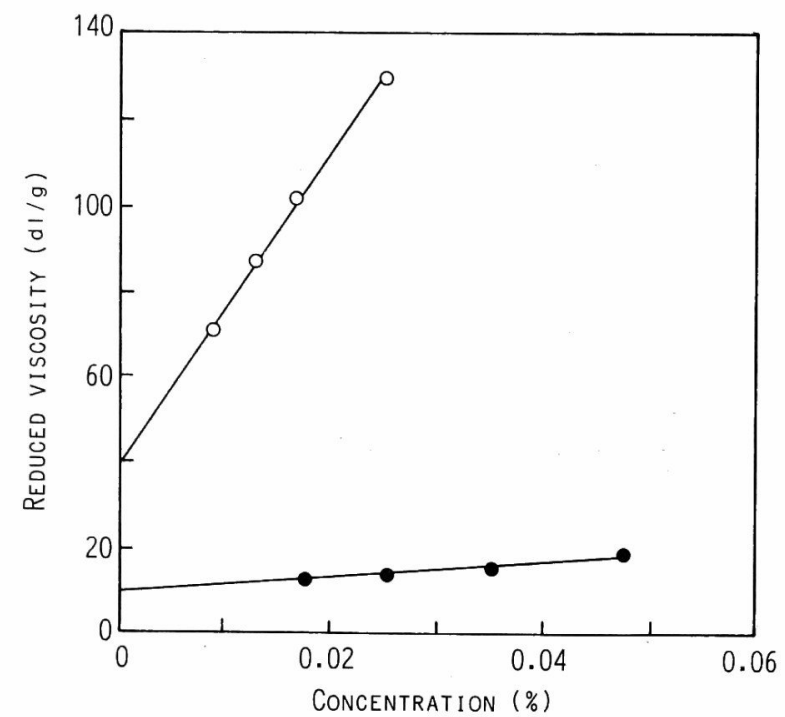

Fig. 5. Intrinsic viscosity of nidamental gland glycoprotein in two different solvents at $25^{\circ} \mathrm{C}$.

The values of intrinsic viscosity were estimated to be $40.0 \mathrm{~d} l / \mathrm{g}$ for water $(\mathrm{O})$ and $10.0 \mathrm{~d} l / \mathrm{g}$ for $0.6 \mathrm{M} \mathrm{NaCl}, \mathrm{pH} 7.2(\bigcirc)$.

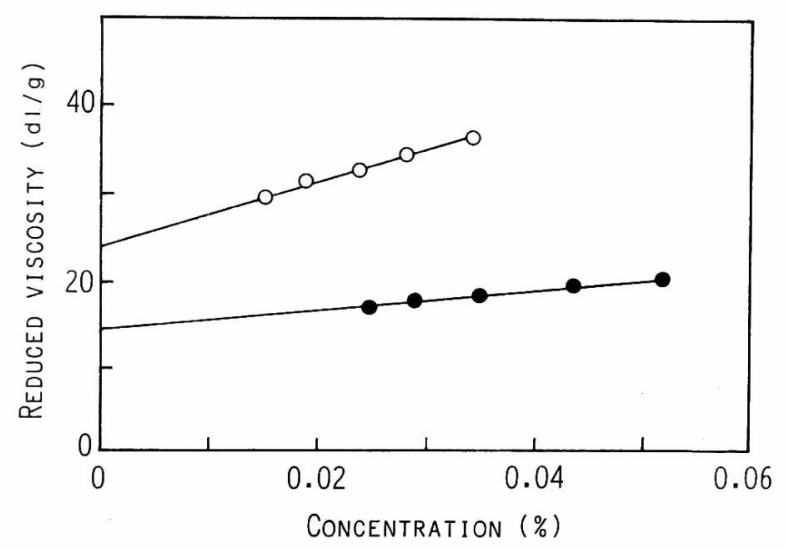

Fig. 6. Intrinsic viscosity of actinase-resistant fragment from nidamental gland glycoprotein.

The values of intrinsic viscosity at $25^{\circ} \mathrm{C}$ were estimated to be $24.0 \mathrm{~d} l / \mathrm{g}$ for water $(\mathrm{O})$ and $14.7 \mathrm{~d} l / \mathrm{g}$ for $0.6 \mathrm{M} \mathrm{NaCl}, \mathrm{pH} 7.2($

$50 \%$ ethanol. The ethanol-precipitate was completely dissolved in $0.6 \mathrm{M} \mathrm{NaCl}$ and thus its macromolecular properties were examined by both viscometry and sedimentation velocity analysis. As shown in Fig. 6, it has an intrinsic viscosity of $14.7 \mathrm{~d} l / \mathrm{g}$ in the solvent of $0.6 \mathrm{M} \mathrm{NaCl}$ containing $0.02 \mathrm{M}$ phosphate buffer, $\mathrm{pH} 7.2$. In the same solvent, its sedimentation pattern reveals a hypersharp peak having a sedimentation constant of $10.5 \mathrm{~S}$, together with a trace amount of faster-moving peak (Fig. 7), indicating the considerably high homogeneity of this precipitate. The high viscosity and the hypersharp peak observed in Figs. 6 and 7 , respectively, are due to the extreme asymmetry of the actinase-resistant fragment in solution. From the equation of Simha ${ }^{14)}$ with the value of $14.7 \mathrm{~d} l / g$ for intrinsic viscosity, the axial ratio of the fragment as a prolate ellipsoid of evolution was calculated to be about 210. Moreover, the molecular mass was calculated to be about $1400 \mathrm{kDa}$ from

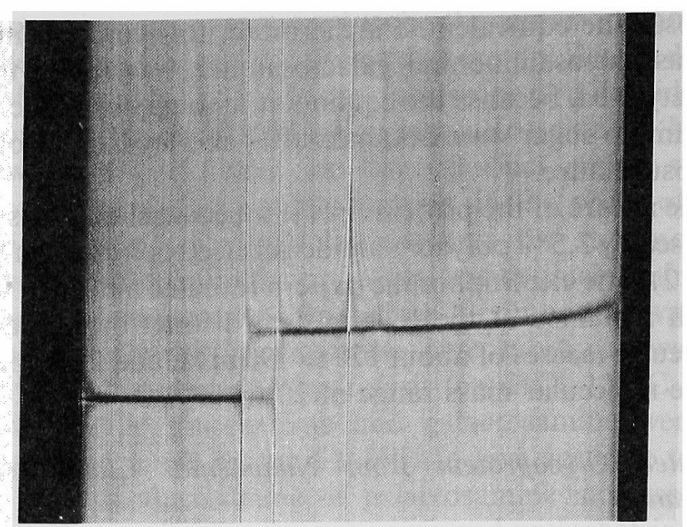

Fig. 7. Sedimentation velocity pattern of actinase-resistant fragment from nidamental gland glycoprotein at $25^{\circ} \mathrm{C}$.

The fragment was dissolved in $0.6 \mathrm{M} \mathrm{NaCl}, \mathrm{pH} 7.2$, at a concentration of $0.045 \%$ and the photograph was taken $13 \mathrm{~min}$ after the speed of $50,000 \mathrm{rpm}$ was reached.

the Scheraga-Mandelkern equation ${ }^{15}$ ) with a value of $3.42 \times 10^{6}$ for $\beta$. On the SDS-polyacrylamide gel, as expected, this large fragment fails to penetrate the gel (Fig. 2).

The $1400 \mathrm{kDa}$ fragment consisted of $18.7 \%$ protein, $76.4 \%$ carbohydrate, and $4.9 \%$ sulfate by weight and thus the masses of protein, carbohydrate, and sulfate in the fragment were calculated to be 260,1070 , and $70 \mathrm{kDa}$, respectively. As a whole, this chemical composition resembled that of the original glycoprotein. Actinase digestion, however, caused a slight increase in the threonine, proline, and isoleucine contents but a marked decrease in those of all the other amino acids except methionine. The fragment was quite unique in the extremely high levels of threonine, proline, and isoleucine which accounted for nearly $90 \%$ of the total amino acid residues. Threonine residues, especially, dominated the amino acid composition, comprising $45 \%$ of the total amino acids. Moreover, the molar ratio of threonine and $\mathrm{N}$-acetylgalactosamine was $1: 1$, indicating the possibility that all threonine residues were attached to $N$-acetylgalactosamine by $O$-glycosidic linkages. The content of ester sulfates was 81 residues $/ 1000$, comprising $11.5 \%$ of the total sugar residues.

\section{Discussion}

In the present study, a sulfated glycoprotein was prepared from squid nidamental glands by solubilization with $0.4 \mathrm{~N}$ $\mathrm{NaOH}$, followed by precipitation with $50 \%$ ethanol, and is apparently distinguished from proteoglycans by the absence of uronic acid residues. We assume that this glycoprotein is defined as a mucin, because a high percent of its weight $(73 \%)$ consists of carbohydrates, $O$-linked to threonine residues in the protein backbone via $N$-acetylgalactosamine residues. ${ }^{13)}$ Although direct evidence for the existence of Thr-GalNAc linkages has not been obtained yet, the compositional nature strongly supports our assumption.

All vertebrate mucins so far examined have been shown to be positive for the modified Morgan-Elson reaction ${ }^{6}$; the method combines the susceptibility of $N$-acetylgalactosamine terminating $O$-glycosidically linked oligosaccharides to $\beta$-elimination with the Morgan-Elson reaction ${ }^{16)}$ 
for $\mathrm{N}$-acetylhexosamines with free reducing ends. As described in this study, the nidamental gland mucin of squid is unusual in that it was resistant to $\beta$-elimination and was negative for the modified Morgan-Elson reaction. This feature is presumably due to some unique amino acid sequence around threonine residues. Such a situation was previously reported for spisulan, a mucin-type glycoprotein from a ground clam Cyprina islandica. ${ }^{17)}$

The nidamental gland mucin was rendered soluble in water by the alkaline treatment and exhibited the extremely high intrinsic viscosity of $40 \mathrm{~d} l / \mathrm{g}$, which is presumably a minimum value because of the relatively high velocity gradient $\left(1000 \mathrm{~s}^{-1}\right)$ of the viscometer used. The addition of a small amount of $\mathrm{NaCl}$ to the mucin solution resulted in a marked decrease of viscosity and the formation of slight turbidity; these are interpreted as being mainly due to the aggregation of mucin molecules, thus it is very defficult to estimate their molecular weight and dimensions.

On the other hand, proteolytic digestion of the mucin molecules with actinase yielded a large peptide fragment which was soluble even in the presence of $\mathrm{NaCl}$. The actinase-resistant large fragment corresponded to about $85 \%$ of the original mucin by weight and was calculated to have a molecular weight of approximately 1,400,000 and to contain $18.7 \%$ protein. Extending these calculations assuming that the protein backbone has a molecular weight of 260,000 , that all threonine residues are $O$-glycosylated, and that $4.9 \%$ of the total weight consists of sulfate, one can calculate that the large fragment contains $1170 \mathrm{O}$-linked oligosaccharide chains, with an average length of 5.3 residues and 3 sulfates per $5 O$-linked chains. Moreover, the molar ratio of threonine, proline, and isoleucine was found to be $2: 1: 1$ and the sum of these amino acids accounted for nearly $90 \%$ of the total amino acid residues. Thus the protein backbone must have some repeating sequences such as T-P-T-I. From these combined results, there is no doubt that the abundance of closely packed $O$-linked glycans protects a large part of the mucin molecule against actinase.

Once secreted, the nidamental gland mucosubstance of squid forms a highly hydrated gel comprising a large number of constituents to offer more perfect protection for eggs. The present study has shown that the most prominent macromolecule in this gel is the mucin-type glycoprotein, which is responsible for the viscous and gel-forming properties of nidamental gland mucosubstance. In order to obtain further information concerning network structure of the gel, more detailed structural studies of the nidamental gland mucin and other constituents are now in progress.

\section{References}

1) R. D. Durward, E. Vessey, R. K. O'Dor, and T. Amaratunga: Reproduction in the squid, Illex illecebrosus: First observation in captivity and implications for the life cycle. ICNAF Sel. Papers, 6, 7-13 (1980).

2) M. Okiyama and S. Kasahara: Identification of the so-called "common squid eggs" collected in the Japan Sea and adjacent waters. Bull. Jap. Reg. Fish. Res. Lab., 26, 35-40 (1975).

3) R. K. O'Dor and N. Balch: Properties of Illex illecebrosus egg masses potentially influencing larval oceanographic distribution. $N A F O S C i$. Coun. Studies, 9, 69-76 (1985).

4) F. J. Oelshlegel, Jr., J. R. Schroeder, and M. A. Stahmann: Simple procedure for basic hydrolysis of proteins and rapid determination of tryptophan using a starch column. Anal. Biochem., 34, 331-337 (1970).

5) C. J. M. Rondle and W. T. J. Morgan: Determination of glucosamine and galactosamine. Biochem. $J$., 61, 586-589 (1955).

6) V.P. Bhavanandan, M. Sheykhnazari, and H. Devaraj: Colorimetric determination of $N$-acetylhexosamine-terminating $O$-glycosidically linked saccharides in mucins and glycoproteins. Anal. Biochem., 188, 142-148 (1990).

7) T. Imanari, Y. Arakawa, and Z. Tamura: Gas chromatographic analysis of aldoses. Chem. Pharm. Bull., 17, 1967-1969 (1969).

8) T. Bitter and H. Muir: A modified uronic acid carbazole reaction. Anal. Biochem., 4, 330-334 (1962).

9) G. W. Jourdian, L. Dean, and S. Roseman: The sialic acid. XI A periodate-resorcinol method for the quantitative estimation of free sialic acids and their glycosides. J. Biol. Chem., 246, 430-435 (1971).

10) T. T. Terho and K. Hartiala: Method for determination of the sulfate content of glycosaminoglycans. Anal. Biochem., 41, 471-476(1971).

11) M. S. Scawen and A. Allen: The action of proteolytic enzymes on the glycoprotein from pig gastric mucus. Biochem. J., 163, 363-368 (1977).

12) S. Kimura, Y. Takema, and M. Kubota: Octopus skin collagen: Isolation and characterization of collagen comprising two distinct $\alpha$ chains. J. Biol. Chem., 256, 13230-13234 (1981).

13) G. T. Strous and J. Dekker: Mucin-type glycoproteins. Crit. Rev. Biochem. Mol. Biol., 27, 57-92 (1992).

14) R. Simha: The influence of Brownian movement on the viscosity of solutions. J. Phys. Chem., 44, 25-34 (1940).

15) H. A. Scheraga and L. Mandelkern: Consideration of the hydrodynamic properties of proteins. J. Am. Chem. Soc., 75, 179-184 (1953)

16) W. T. J. Morgan and L. A. Elson: A colorimetric method for the determination of $\mathrm{N}$-acetylglucosamine and $\mathrm{N}$-acetylgalactosamine. Biochem. J., 28, 988-995 (1934).

17) J. A. Cifonelli and M. B. Mathews: Structural studies on spisulan: A mucopolysaccharide from clams. Conn. Tissue Res., 1, 231-241 (1972). 